\title{
Forensic Interview of Sexually Abused Children: The Case of Three Selected Child Protection and Investigation Units in Addis Ababa Ethiopia
}

\author{
Tensae Gebrekrstos Gebreegziabher \\ Lecturer of Law and policing, Ethiopian Police University College
}

\begin{abstract}
This study examines the application of forensic interview methods used in police investigations to gather evidence from sexually abused children. The investigating police officers need a range of skills related to interviewing victims, helpful in the course of detecting suspects. An effective interview is therefore a milestone to the investigating officers, plan a new way of eliciting relevant information from additional sources. Typically, interviewing requires the police to attempt to identify the type of sex crime considering the phases of pre, present, and post crime acts. The study used a qualitative method to explore the opinions of informants in depth. Seventeen participants were drawn from seven investigating police officers, three public prosecutors, three social workers, and four administrators selected using a purposive sampling technique. Data pertinent to the study were gathered using unstructured and key-informant interview techniques. Besides, observation and document reviews were employed to complement the data solicited from both sources. Thematic analysis was applied to give a thick description of data opinionated by informants. The study shows that investigating police officers are poorly acquainted with the interview techniques established by the Federal Supreme Court Interview Techniques Guideline. The guideline explicitly advocates the police officers' use of free narrative open-ended questions implying ample room to the child to describe the situation of the abuse in his/her own words. The study conveys the message that the police officers should take a series of training on forensic interviews and design the landscape where regular feedback, supervision, and stress management mechanisms will regularly be exchanged.
\end{abstract}

Keywords: Child Sexual Abuse, Forensic Interview, Questioning Techniques, Challenges

This is an open access article under the CC-BY-NC license.

\section{INTRODUCTION}

Ethiopia is one of the countries endorsed the Convention on the Rights of the Child (CRC) and the African Charters on the Right and Welfare of the Child (ACRWC). The CRC was adopted by the United Nations (UN) in 1989 while Ethiopia became a signatory in 1991. One of its main principles is "the [child's] right to be heard and to express views and concerns" (CRC, Article 12). In particular, children need to have the opportunity to be heard in any judicial or administrative proceedings including police investigations. The CRC recommends the opinion of children be weighed by their age and maturity. Interviewing sexually abused children is considered the most challenging one in the proceedings of police investigations. The impact caused by this will finally lead the investigating officers to determine their views on the degree and level of abuse. One of the reasons is due to the insensitive nature of police forensic interviewers (Harris, 2010; La Rooy, Lamb \& Memon, 2011).

Due to the unprecedented results of interviewing sexually abused children and the challenges of a forensic interview, considerable efforts were laid to maintain the technical capacity and knowledge of police in gathering evidence from victims' point of view. Children victims of different types of crime require lengthy periods to fully recover from their traumatic experiences. However, letting them 
describe their feeling about the crime and the harm that they sustained helps the police officers to sympathize with the problem and alter different ways of gathering evidence admissible to adjudication. Several studies carried out on child sexual abuse finally reported that it is done with a carefully designed strategy of silence and being a witness -free crime, often leaving no physical signs and actively hidden by wrongdoers (Cronch et al., 2006; Harries, 2010; Jibril, 2012). Hence, disclosure of sexual abuse through proper interviewing techniques is key to providing support and arranging appropriate further interventions to treat a child victim of abuse. When children do not volunteer information or delay disclosure, the possibility of continued victimization is magnified and it becomes more difficult to design appropriate future interventions (Cronch et al., 2006; Harries, 2010). Usually, an investigator should encourage a child alleged of sexual abuse or witness to recall what happened and explain the incident in her/his own words (UNODC, 2009, p.15).

Ethiopia formulated various schemes in developing frameworks and standards and improved institutional arrangements to fiercely fight the offenders and help sexually abused ones. The Constitution of the Federal Democratic Republic of Ethiopia (FDRE Constitution) devotes a full article on the rights of a child. The constitutional provision embraces, inter alia, the precept of the consideration of 'the best interest of the child' in every matter concerning children undertaken by public and private institutions (FDRE Constitution, 1995, Art. 36). But the realization of both the principle of "the best interests of the child" and "the right to be heard and to express views and concerns" regarding sexually abused child remains largely hidden in the criminal justice system, particularly in the police investigations. Child victims of sexual abuse continue to suffer from feelings of suspicion, stigma, betrayal, and powerlessness for the rest of their lives. Above all, sexually abused children suffer the second traumatization as a result of insensitive criminal justice institutions including police investigations perpetuates these feelings (Getachew, 2011; Jibril, 2012; Tsegaye, 2011).

There is no research conducted on forensic interview practice with sexually abused children in Ethiopia. Hence, this particular study is aimed to specifically examine the application of child forensic interview techniques guidelines and challenges that stalled effective forensic interviews of sexually abused children in Gandhi Memorial Hospital, Arada, and Yeka Women and Children Protection and Investigation Units.

\section{LITERATURE REVIEW}

The forensic interview does not have one definition that works out all over the world. Dune (2006) define forensic interview as a semi-structured, one time videotaped interview of the child by a trained professional, provided that there has been a disclosure of sexual abuse or there is a reason or strong suspicion to believe the child has been sexually abused based upon behavioral observation or medical evidence. This narrow definition seems very as a forensic interview is a wide concept that involves more than the child victims of sexual abuse. Similarly, North Carolina's Division of Social Services and N.C. Family and Children's Resource Program (2002) defines the term forensic interview as a technique used to obtain a statement from a child in an objective, developmentally sensitive, and legally defensible manner. Swerdlow-Freed (2009) defines a forensic interview as structured conversations with a child- victim, and witnesses of both physical and sexual abuses to elicit an accurate account of events. This definition is quite helpful to understand forensic interviews' worth for all victims of physical, sexual abuse, and witnesses of such abuse. A forensic interview is decisive in producing evidence admissible in criminal prosecution. Forensic interviews are legally sound, in part, because they ensure the interviewer's objectivity, employ non-leading techniques, and emphasize careful documentation of 
International Journal of Management, Entrepreneurship, Social Science and Humanities (IJMESH), Vol. 3 (1), 67-79

Forensic Interview of Sexually Abused Children: The Case of Three Selected Child Protection and Investigation Units in Addis Ababa Ethiopia

Tensae Gebrekrstos

the interview (Harris, 2010; North Carolina's Division of Social Services and the N.C Family and Children's Resource Program, 2002). Newlin et al. (2015) defined the term forensic interview as:

A forensic interview of a child is a developmentally sensitive and legally sound method of gathering factual information regarding allegations of abuse or exposure to violence. This interview is conducted by a competently trained, neutral professional utilizing research and practice-informed techniques as part of a larger investigative process (p.3).

Hence, the concept of a forensic interview is broader than child forensic interviews and even broader than forensic interviews of sexually abused children is. Various scholars and institutions use the term forensic or investigative interview differently to refer to the interview of sexually abused children. For this particular study, a forensic interview of sexually abuse children refers to a structural conversation designed to obtain information or evidence from the children about the sexual abuse she/he experienced.

Learning the methods and techniques of carrying out child forensic interview undoubtedly help police officers acquire knowledge and skill to effectively and appropriately conduct forensic interviewing. As a result, experts in a forensic interview used previous research findings to develop the guidelines for interviewers. The most influential and greatly adopted standard comes from the National Institute of Child Health and Human Development (NICHD) interview guideline (Cronch et al., 2006; Faller, 2015). According to the International Society for Prevention of Child Abuse and Neglect (ISPCAN), the NICHD mainly to encourage forensic interviewers to use open-ended prompts in extracting information or evidence from the child alleged of sexual abuse (ISPCAN, 2011). As La Rooy et al., (2015) described, it is the most common interview protocol developed about child development issues including linguistic abilities, memory retrieval capacities, suggestibility in interviewer's behavior, and effect of stress and trauma. Studies conducted by Pipe, Orbach, Lamb, Abbott, and Stewart (2013) witnessed the effects of the NICHD protocol for interviewing sexually abused children on case outcomes and indicated that children interviewed with the NICHD protocol provide significantly more details and the protocol was found to be equally effective for all ages. Numerous studies showed that ongoing training, supervision, peer reviews and other forms of feedback should help forensic interviewers to integrate the skills they learned during initial training and also improve their practice through time (Faller, 2015; Lamb et al., 2007; Newline et al., 2015). Based on these studies, the NICHD interview protocol guides the police investigative interviewer to apply the various stages of legally sound interview techniques; mainly varying from highly structured questions to semi-structured to flexible ones. The most reliable information is obtained when a continuum of questions are used.

Better investigative interviewing by the police is important to ensure the protection of the sexually abused children and the conviction of wrongdoers. To this end, a range of forensic interview guidelines and specific questioning techniques of sexually abused children received ample room in various literature. Several studies investigated forensic interview guidelines and questioning techniques of sexually abused children (Cronch et al., 2006; Faller, 2015; Smith, 2014). The studies found shortcomings within police investigations and recommended the existence of well-entrenched guidelines to ease the trial process in the justice system.

Another study in New Zealand examined the common understandings and challenges faced by child forensic interviewers (Wolfman, 2016). Lack of access to regular feedback, supervision, guided selfreview was among the listed challenges. Similarly, in Scotland, quantitative research by La Rooy et 
la.,(2011) studied the adherence of police forensic interviewers to the Scottish Executive Interview Guideline. The study found that forensic interviewers mostly adhered to the guideline however interviewers need feedback on their interview process. Wood and Garven (2000) also studied clumsy interviewing and it's the implication for police forensic interviewers as a result of failure to use recommended interviewing techniques. Clumsy interviews may occur even with highly trained and experienced interviewers due to forgetfulness, lack of skill, and lack of supervision, and this in turn results in a lack of detail in children's responses, reduced credibility of children's statements and thereby reduced likelihood of conviction. The study recommended that forensic interviewers should have experience in interviewing children; be trained and supervised to avoid improper interviewing techniques.

To the best of the researcher's knowledge, little is done towards proper forensic interviews and its application in the police investigation. Lack of the interviewing techniques of children victims of various crimes is therefore unstudied when it comes to a plan targeted to improve the police investigation. Because the interview held with children does not easily align with the basic procedures to be used in the time of gathering evidence related to the crime. Evidence submitted in court is often not sufficient to explicitly show the crime. It mainly arises from a lack of skill in both processing crime scene, gathers evidence, and exhibits evidence in police stations. These all sabotaged the trial process and have been a cause for the files to be closed in a trial process. This study is therefore in place to probe the factors that are untouched and fill the gaps in contributing some on the existing body of knowledge towards the interview techniques of sexually abused children.

\section{RESEARCH METHOD}

A qualitative research design was used. A qualitative design was chosen due to its flexible nature, helpful for understanding real-life contexts and allowing the active involvement among the study participants (Creswell, 2007, p.40). Yin (2011) also confirmed that qualitative research allows the researcher to explore the identified social problem in its social context. In this study, the researcher assumes the participants provide the meanings of their experiences against the application of forensic interview techniques guidelines and the challenges they face as well. .

This study relied on the case study method as it allows the possibility of gaining significant knowledge about the issue under scrutiny. Notwithstanding, the study was exploratory since exploratory case study was applied: to explore entirely new fields of research when the researcher has only a few or no antecedents as far as explaining the focused phenomenon (Yin, 2003). This research site is also selected purposefully because the inquirer selects individuals and sites for study. After all, it can purposefully inform an understanding of the research problem and the central phenomenon of the study (Creswell, 2007). For this reason, this study used an exploratory case study approach.

As Creswell (2007) stated, in qualitative research, the major tools of data collection include an interview, observation, and document review. Data collection is a series of interrelated activities aimed at gathering good information to answer the research questions. Hence, proper case study design involves detailed, in-depth data collection involving multiple sources of information-rich in context (Creswell, 2007; 2014). As Yin (2003) stated, "data for case studies may come from documents, archival records, interviews, direct observation, and physical artifacts" (p. 86). In this study, both primary and secondary sources were used to gather pertinent data. In-depth interviews and observation were used to collect primary data while secondary sources such as written documents, including the internationally 
recommended Investigative Interview Techniques Guideline adopted by the Ethiopian Federal Supreme Court Child Project Office (2009) was also used.

\section{RESULTS AND DISCUSSION}

Discussion of the results and begin by reporting the application of Federal Supreme Court Child Project office Investigative Interview Techniques Guideline (2009). The study assessed the police forensic interviewers' awareness and application of the standards presented in the guideline and the challenges encountered in carrying out extensive interviews with sexually abused children.

\section{Awareness on the Interview Techniques Guideline} Introduction

According to the findings of the study, informants confirmed that they are well aware of the guidelines advising them to start by introducing the reason why the interview is important. Likewise, the interviewees are well acquainted with the benefits that the guideline advocates on the importance of introducing with victims to establish a long-lasting relationship. Their understanding seemingly complies with the investigative interview guideline that insists police forensic interviewers to introduce their name, role, place of work, method of documentation and confidentiality (Federal Supreme Court Interview Techniques Guideline, 2009, pp.2-4).

However, as the finding indicates, the interviewer fails to explain the most crucial points highly magnified in the guideline: neither tell the child about the documentation methods that they are going to use and its importance nor explain the ground rules of the interview. Besides, the interview is rightly conducted in the presence of public prosecutors and social workers formed as a team to detect the case of sexually abused children. For privacy reasons however the guideline suggests police investigative interviewers conduct the interview alone only if it found important (pp.2-4).

\section{Rapport building}

The results of the study indicated that the interview with victims commence after making sure the rapport is built. They agreed that the investigating officers attain a relative advantage if they could explain victims from the child's developmental stage, communication capacity and language competence of the child to conduct an interview. Typically, they invite the child to talk about issues that are not linked with the abuse but events that lead to the abuse. Questions like 'Tell me about things you like to do.' Where do you go to school? What grade are you in? What is the name of your teacher? Do you like school?" What are the things you like best about school? are among the rapport building questions that lead the child to feel comfortable with the interview process. Establishing a rapport before moving on to more sensitive questions also helps the interviewer to obtain preliminary information about child verbal skills and cognitive development. According to the interview guideline, interview questions like 'Tell me about things you like to do' 'I want to know you better' 'I need you to tell me about the things you like to do' 'Tell me more about that' These questions are not directly linked to the targeted issue but events that lead the child to tell about the actual happening (p.5).

The in-depth interview informants also believe that building rapport help to put the child victim at ease and this in turn creates the opportunity for interviewers to gain the children's willingness to communicate. Similarly, as (La Rooy et al., 2015, pp.10-11) stated in the rapport-building phase the child alleged of sexual abuse begin to trust the interviewer and become cooperative in the interview process. 
Rapport building begins with the initial introduction and continues throughout the interview process. However, the informants believe that rapport building should come before the interview.

\section{Training in episodic memory}

The findings of the study confirmed that the informants are well aware of what training in episodic memory/narrative event practice is. They asserted that it is a neutral topic worthy of embracing to ensure the application of the first phase of rapport building is suggested to become ahead of the substantive issues are discussed. This undoubtedly helps the child to become more aligned with the required processes of answering open-ended questions. Before the interview, forensic interviewers motivate the child to say the events he experienced in recent times; such as the first day of school, birthday party, holiday, and other special occasions that the child can remember. Besides, they even ask the child to describe the events that took place at the same time as the alleged sexual abuse in his own words. This is congruent with the investigative interview guideline where the interviewer is required to identify recent events the child can remember (pp.5-9). Similarly (Faller, 2015; La Rooy et al.,2015) stated this phase is a continuation of the rapport building phase where forensic interviewers also learn more about the communicative abilities of the child alleged of sexual abuse and prepare the child to respond in the substantive phase of the interview. Nevertheless, few participants didn't distinguish the difference between rapport building and the phase of episode memory. Rather, they believe that rapport building and narrative event practice phases are similar in assuming both are in place for the sake of establishing a good relationship.

\section{Transition to substantive issues}

One of the stages of the investigative interview guideline is introducing the topic of abuse. According to the finding, participants agreed that the transition to substantive issues begins when the interviewer prompts a transition to the targeted abuse. Questions such as "would you tell me the reason why you're here today? Would you inform me of the details that happened in this regard?" or someone informed me what wrong went on? Tell me everything about that?" are the one from other interviewers are required to ask the child. Consistent with this finding, the investigative interview guideline suggests interviewers ask the child similar questions as the research participants revealed. As per the guideline, questions that lead to substantive issues include:

Now that I know you a little better, I want to talk about why [you are here] today. I understand that something may have happened to you. Tell me everything that happened that day from the beginning to the end. As I told you, my job is to talk to kids about things that might have happened to them. You must tell me why [you are here/ you came here/ I am here]. Tell me why you think [your mum, your dad, and your grandmother] brought you here today [or 'why you think I came to talk to you today]... (pp.9-14).

Though most of the in-depth informants identified most of the questions to ask during this phase of the interview, few of them believe that the type of questions to ask depends upon the response of the child. 


\section{Investigating the incidents}

Almost all the informants use questions that encourage the child to talk more about the abuse with open-ended questions. Participants also believe that considering the age and communicative level of the child is the first thing to consider in posing open-ended questions during this phase. To the indepth informants, open-ended questions include "Tell me everything that happened." Tell me everything from the beginning to the end." "You mentioned.... Tell me everything about that." "Then what happened?" These types of questions according to the participants help the child to tell a detailed story of the abuse.

Similarly (Faller, 2015; Lamb et al., 2007; La Rooy et al., 2015) believe that open-ended questions usually encourage children to describe events in their own words. Open-ended questions allow children to select the specific details they want to discuss and encourage multiple-word responses. This includes questions like what happened. Tell me everything you can remember? Tell me everything from the start to the end?

Likewise, the investigative interview guideline suggests open-ended questions that help the children to describe the abuse in their own words. This includes:

Tell me everything about that; then what happened? Think back to that [day/night] and tell me everything that happened from [some preceding event mentioned by the child] until [alleged abusive incident as described by the child]. Tell me more about [person/object/ activity mentioned by the child]. You mentioned [person/ object/ activity mentioned by the child], tell me everything about that... (pp.15-20).

On the other hand, though few participants believe that open-ended questions are good as they encourage the child to talk about the sexual abuse, they were unable to tell the proper types of questions to ask then questions that begin with who, what, where, when and why.

Break

Almost half of the in-depth informants practice taking a break during the interview process. This is, in particular, common when the interview is taken place for long hours. During the break time, the police along with the prosecutors and social workers review the information received, look missed information, and plan for the rest of the interview including framing relevant interview questions. Similarly, the guideline stated that interviewers require considering break that helps her/him to revise the information received, to fill the forensic checklist, determine if important information missed, plan and prepare interview questions for the rest of the interview process (pp.20-21).

On the other hand, the rest of the in-depth informants do not take breaks in their interviews. Even when the interview is taking for long hours they continue to ask the child. Because they believe that taking breaks may result in interruption of the flow of the interview and may even lose forensically important evidence. This is contrary to the investigative interview guideline that requires forensic interviewers to take breaks in the interview process.

\section{Gathering additional information not mentioned by the child}

Form the findings of the study few of the in-depth informants are familiar with the type of questions to ask when important information/evidence is missed. They prompt the child by indicating some important issues like an object, activity, place, and feeling mentioned earlier by the child. When has 
the child begun to talk about the missed information they follow the common open-ended prompts such as 'tell me all about that? Likewise, the investigative interview guideline requires interviewers to ask some probing questions but only after exhausting open-ended questions and whenever forensically important questions are missed (pp.21-22). The rest in-depth informants have awareness about the types of interview questions when there is information the child did not tell. They mostly ask openended invitations to help the child disclosed in her or his own words. They also indicate some previously told information to help the child remember the fact.

\section{If the child fails to mention essential information}

As the findings show, almost half of the in-depth interview informants are partly aware of the type of questions when the child fails to mention the information they expected to be answered. They pose some specific questions that help them to link with the previously obtained information or evidence. The guideline demands interviewers to ask some only relevant prompts. Above all, the type of question to ask varies depending upon the response of the child as well as the knowledge of the interviewer regarding the abuse and prior disclosure made by the child (pp.23-25).

Conversely, the rest in-depth informants pointed out that they try to convince the child whatever interviewing questioning techniques including forced-choice ones. Besides, they don't even follow standardized questioning techniques to take information from the child victim.

\section{Information about the disclosure}

Most of the in-depth interview informants stated that they follow different approaches depending upon the response of the child. They commonly ask questions such as "who did the child initially tell? Who else knows what happened?" "You've told me why you came to talk to me today. You've given me lots of information and that helps me to understand what happened." However, rather than listing the types of questions, they ask they didn't identify the specific options mentioned in the guideline. According to the guideline, there are two options to follow during this phase. If a child has mentioned telling someone about the abuse, the interviewer should ask "Tell me everything you can about how ["the first person mentioned by the child"] found out.' Whereas if the child has not mentioned telling anyone, probe about possible immediate disclosure by saying: 'Tell me what happened after [the last incident].' 'And then what happened?' In general, the interviewers should use appropriate interview questions depending upon the response of the child (pp.25-27).

However, few in-depth-interview informants were even unable to mention the specific question to ask during this phase. They don't even have any specific technique to follow than asking every possible question that helps the child to disclose. In sum, almost all of the in-depth interview informants are not fully aware of this phase.

\section{Closure}

According to the findings of the study, almost all of the in-depth interview informants were aware and implementing the closure phase of the forensic interview process. They ask the child if something else has left to tell regarding the abuse. Further, they thank the child for participating in the interview process and the information the child fed them. And, if the child needs some treatment they refer the child to the social worker to help the child feel safe before leaving their office. In a similar vein, the guideline demands investigative interviewers ask the child if something has been left out, thank for the 
information the child told them, and give some counseling service for the child if she/he is yet in need of some treatment (p.27).

However, few interviewers are not fully aware of the closure phase of the interview and they didn't reveal the possible issues to mention under this phase. The only thing they told the researcher about the closure phase is that they simply ask the child if something left to tell and thank the child without addressing further issues covered in the guideline.

\section{Challenges of forensic interview practice}

The study also examined major challenges that hinder effective forensic interview practice of sexually abused children in the study settings. Lack of training, lack of feedback and supervision on the forensic interview practice, lack of stress management mechanism, improper forensic interview room settings, turnover of forensic interviewers, and lack of multidisciplinary team approach were reported as major challenges of forensic interview practice.

\section{Lack of training}

The participants revealed that lack of regular is one of the main challenges that forensic interviewers face in interviewing sexually abused children. Even some of the police officers are employed without having initial training on how to investigate sexual abuse cases. The only training they get is general police science training and some other on the job training that doesn't even directly enable them to conduct a forensic interview. However, the study by Faller (2015) showed that countries that adopt the NICHD Protocol have trained their forensic interviewers. The training mainly ranges from two days to one week. A worldwide study by La Rooy et al., (2015) also indicated that forensic interviewers such as police and social workers are highly trained to interview sexually abused children. Further, the UN Guidelines on Justice in Matters involving Child Victims and Witnesses of Crime (2009, Art.13) require justice personnel should train in dealing with sexually abused children. The training mainly took five to ten days. The training inter alia includes familiarizing forensic interview guidelines, practicing interviews with trained individuals, receiving regular feedback on practice interviews, learning about communicative, and child developmental issues.

\section{Lack of feedback and supervision}

The participants also revealed that there is neither feedback nor supervision mechanism on the interview they conduct. Consistently, finding of the studies by Korkman (2006) in Finland and Wolfman (2016) in New Zealand indicated that there are challenges of regular feedback and supervision that forensic interviewers face in both countries. The study noted that regular feedback and supervision of police on the interview they conduct ensures their adherence to the specific interview guideline they follow and enhance their skill of interview. Nonetheless, the studies have also revealed the existence of some ongoing feedbacks and supervision in these countries. Similarly, the study by (La Rooy et al., 2015) found that the existence of one investigative interviewer in some police departments does not have the chance to give feedback. The studies noted that feedback enables forensic interviewers to deliver better service to the child victim, reduce further abuse, and protect innocent individuals from criminal punishment. The major cause for the absence of forensic interview supervision or guidance as the participants stated is a lack of experienced professionals and lack of commitment by the concerned institution. 


\section{Lack of stress management mechanisms}

Lack of stress management mechanisms was another major challenge where most respondents replied. Traumatic nature of job together with the excessive workload results in police investigative officers facing stress and burnout. However, as the research participants revealed no stress management mechanism helps the interviewers to overcome the challenge and thereby lead a stronger workforce. In line with this, Chiarelli-Helminiak (2014) mentioned that forensic interviewers of sexually abused children are vulnerable to job-related stress. This in turn affects the child victim and the organization they work in. The major cause as Chiarelli-Helminiak stated includes a lack of support from supervisors and coworkers, a lack of job satisfaction, and excessive workloads.

\section{Inappropriate interview room settings}

The participants stated that the interview room arrangement is not comfortable with victim children to tell their story to the interviewer. The rooms are threatened and not as such equipped with necessary facilities to conduct forensic interviews. The rooms are not friendly and noisy. This was also confirmed by the researcher's observation of the selected areas specifically Arada and Yeka units where the uniformed police interviewed a child victim who came to the office in the presence of other clients. The privacy of the child victim suffered due to people who want to enter the head of the sub-city crime investigation department, head of the unit, and other persons. Likewise, the location of the interview room is attached to the corridor of three offices.

In line with this, the study by Pangborn (2009) stated forensic interviews are commonly conducted in police stations by uniformed police in an unfriendly environment. This may contaminate the interview process. The study by Tsegaye (2011) has also indicated that though there are separate blocks/rooms the units/centers didn't have proper settings and facilities that enhance the childfriendliness of the unit. Similarly, Getachew (2011) studied the challenges of child victims in Ethiopia and found that child victim are subjected to cycles of traumatization during the criminal justice process. The major predicaments include a lack of respect for the privacy of the child victim during the investigation and threatening interview room settings.

\section{Turnover of forensic interviewers}

Findings from the research participants depict that there is a high turnover of forensic interviewers. The participants mentioned salary and per dim differences among different professionals working similar jobs in the selected areas; the traumatic and challenging nature of the job along with the poor working environment is a major cause for the turnover of experienced and trained forensic interviewers. The experienced and skilled interviewers including the police investigative officers, social workers, child psychologists, and prosecutors who know the case very well leave their job and handover the case to the inexperienced individuals. Interviewers as (O'Donohue \& Fanetti, 2015) stated are exposed to traumatic child victims and witnesses of crime that directly affect the turnover of professionals

\section{CONCLUSION}

The study concluded that most of the police forensic interviewers are cognizant with the introduction, rapport building, training in episodic memory, transition to substantive issues, investigating the incident, taking a break, and closure phase of the investigative interview guideline. However, they are neither fully familiar with the remaining phases nor fully aware of most of the 
interview questions stated in each phase of the guideline. Likewise, the interviewers believe that narrative or open-ended questions are preferable as they give the chance to the child to describe the abuse by her/her own words. But most of the time they tend to get information quickly by sticking on the leading or suggestive ones. They believe that open-ended questions are time-consuming and difficult to utilize since there is pressure to obtain evidence quickly for the court.

Lack of forensic interview training, lack of feedback and supervision on forensic interview practice, lack of stress management mechanism, improper forensic interview room settings, and turnover of forensic interviewers are among the major challenges that stalled effective forensic interview practice. Accordingly, the investigative police officers need urgent training of forensic interview techniques and this should be accompanied by continuous feedback and supervision. There should be a separate unit/room, as in the Gandhi Memorial Hospital, to deal with child abuse cases including a forensic interview. A child forensic interview is a traumatic and overburden job, there should be stress management mechanisms and thereby retain the excessive turnover of the interviewers in the selected settings.

\section{REFERENCES}

Chiarelli-Helminiak,C.,M. (2014). Organizational factors affecting job satisfaction and burnout among forensic interviewers (Doctoral Dissertations). University of Connecticut. Retrieved from http://opencommons.uconn.edu/dissertations/397/

Creswell, J. W. (2007). Qualitative inquiry and research design: Choosing among five approaches (2nd Ed.). California: Sage.

Creswell, J.W. (2014). Qualitative, quantitative and mixed methods approaches (4thed.).Sage.

Cronch, L.E, Viljoen, J.L., Hansen \& D. J. (2006). Forensic interviewing in child sexual abuse cases: Current techniques and future directions. University of Nebraska-Lincoln: Elsevier 11(3), 195207.doi:10.1016/j.avb.2005.07.009

Dunn, S.E. (2006). Interviewing pre-school age victims of child sexual abuse: interviewing methods \& disclosure outcomes (master's thesis). Georgia State University. Retrieved from http://scholarworks.gsu.edu/psych_theses/13

Faller,C. K. (2015). Forty years of forensic interviewing of children suspected of sexual abuse, 19742014:Historical benchmarks, Journal of Soc. Sci.4, 34-65; doi:10.3390/socsci4010034

Federal Supreme Court Child Project Office (2002). Interview Techniques Guideline.

Getachew Assefa .(2011). The predicaments of child victims of crime seeking justice in Ethiopia: a double victimization by the justice process. Journal of Afrika Focus, 24(1), 33-50. Retrieved from ojs.ugent.be/AF/article/view/4993

Harris,.F (2010). Toward a better way to interview child victims of sexual abuse. Palladian Partners. Retrieved from https://www.nij.gov/journals/267

International Society for Prevention of child abuse and Neglect (ISPCAN). ( 2011, October). Comparing the NICHD and RATAC child forensic interview approaches - Do the differences matter? Child sexual abuse. 20(1). 20(1),1-8. Retrieved from docplayer.net/23184313-Comparing-the-nichdand-ratac-child-forensic-interview-appro.

Jibril Jemal (2012). The child sexual abuse epidemic in Addis Ababa: Some reflections on reported incidents, psychosocial consequences and implications. Jimma University. Ethiopia. Journal of Health Sci., 22(1), 59-65. 
International Journal of Management, Entrepreneurship, Social Science and Humanities (IJMESH), Vol. 3 (1), 67-79

Forensic Interview of Sexually Abused Children: The Case of Three Selected Child Protection and Investigation Units in Addis Ababa Ethiopia

Tensae Gebrekrstos

Korkman, J. (2006). How (not) to interview children interviews with young children in sexual abuse investigations in Finland (Master's thesis). Abo Akademi University. Retrieved from http://bibbild.abo.fi/ediss/2006/KorkmanJulia.pdf

Lamb, M. E., Orbach, Y., Hershkowitz, I., Esplin, P.W., \& Horowitz, D. (2007). Structured forensic interview protocols improve the quality and in formativeness of investigative interviews with children: A review of research using the NICHD. Investigative Interview Protocol. Child Abuse \& Neglect, 1(2). doi:10.1016/j.chaibu.2007.03.021

La Rooy, D., Lamb, M. E., \& Memon, A. (2011). Forensic interviews with children in Scotland: A survey of interview practices among police. Journal of police and criminal psychology, 26(1), 1-29. doi: 10.1007/s11896-010-9072-9

La Rooy,D.,Brubacher,S.,Aromäki-Stratos,A.,Cyr,M.,Hershkowitz,I.,Korkman,J.,Myklebust, T., Naka,M., E. Peixoto,C., Roberts,K., Stewart,H.\& Lamb,M.E.(2015). The NICHD protocol: a review of an internationally-used evidence-based tool for training child forensic interviewers. Journal of criminological research, policy and practice, Emerald group publishing, 1(2)2,76-89, http://dx.doi.org/10.1108/JCRPP-01-2015-0001

Newlin,C.,Steele,.L.C.,L.,Chamberlin, A., Anderson, J., Kenniston, J., Russell, A., Stewart,H.,\& VaughanEden,.V.(2015). Child forensic interviewing. Best practices (Bulletin).US Department of Justice. Juvenile justice bulletin,1-17. Retrieved from https://www.ojjdp.gov/pubs/248749.pdf.

North Carolina's Division of Social Services and the N.C. Family and Children's Resource Program.(2002, December). Forensic interviewing and child welfare services in North Carolina 8(1). Retrieved from www.practicenotes.org/vol8_no1/cspn_v8no1.pdf

O'Donohue, W., M.,F.(Eds.).(2015). Forensic interview regarding child sexual abuse: A guide to evidence based practice. New York, NY:Springer.

Retrieved from www.springer.com/gp/book/9783319210964.

Pangborn, K., R. (2009). Identifying and correcting problems with forensic interviews of alleged child sexual abuse victims: A holistic environmental approach. Journal of Vol.18,18-1. Retrieved from www.ipt-forensics.com/journal/volume18/j18_1.htm.

Pipe, M.-E., Orbach, Y., Lamb, M. E., Abbott, C. B., \& Stewart, H. (2013). Do case outcomes change when investigative interviewing practices change? Psychology, Public Policy, and Law, 19(2), 179-190. doi: $10.1037 / \mathrm{a} 0030312$

Smith, S. (2014). Forensic assessment model for the sexually abused child in the South African context (Doctoral dissertation). North-West University.

Retrieved from https://dspace.nwu.ac.za/handle/10394/12263.

Swerdlow-Freed, D., H. (2009). Questioning strategies for forensic interviews of children. Retrivedfrom:https://www.drswerdlow-freed.com/pdf/physical-sexual-

abuse/QUESTIONING\%20STRATEGIES\%20FOR\%20FORENSIC\%20INTERVIEWS\%200F\%20

CHILDREN.2011.pdf

The African Charter on the Rights and Welfare of the Child. (1990).

The Constitution of the Federal Democratic Republic of Ethiopia. (1995).

The Convention on the Rights of the Child. (1990).

The Federal Democratic Republic of Ethiopia (FDRE), Criminal Code. (2004).

The National Institute of Child Health and Human Development (NICHD) Interview Protocol: Retrieved from nichdprotocol.com/NICHDProtocol2.pdf. 
International Journal of Management, Entrepreneurship, Social Science and Humanities (IJMESH), Vol. 3 (1), 67-79

Forensic Interview of Sexually Abused Children: The Case of Three Selected Child Protection and Investigation Units in Addis Ababa Ethiopia

Tensae Gebrekrstos

Tsegaye Deda (2011). Introducing child protection and child friendly justice in a society with complex socioeconomic challenges. Experiences and lessons from Ethiopia.

United Nations Office on Drugs and Crime(UNODC).(2009). Justice in matters involving child victims and witnesses of crime. Model law and related commentary. Vienna, Austria.

Wolfman, M. (2016). Understanding and addressing challenges faced by forensic interviewers in their work with children (Master's thesis). Victoria University of Wellington. Retrieved from esearcharchive.vuw.ac.nz/xmlui/bitstream/handle/10063/5144/thesis.pdf

Wood, J. M., \& Garven, S. (2000). How sexual abuse interviews go astray: Implications for prosecutors, police, and child protection services. Child Maltreatment, 5(2), 109-118. doi:10.1177/1077559500005002003

Yin, R.,K. (2011). Qualitative study from start to finish. New York. The Guilford Press.

Yin, R,K. (2003). Case study research design and methods Applied social research method series (3rd ed.). USA: Sage. 